\title{
Éditorial
}

\section{« Et la lumière fut » : rôle de l'EBM ?}

\section{Jacky Samson}

\author{
Rédaction en Chef
}

En Amérique du Nord, depuis cinq décennies, quelques enseignants cherchent à faire évoluer la technique d'enseignement de la Médecine et sa pratique, et ils ont développé une approche innovante dans ces deux domaines. Dans les années 1960, ils ont proposé de remplacer l'enseignement linéaire par l'enseignement intégré par appareil ou par organe (c'est-à-dire enseignement dans le même temps de l'embryologie, l'anatomie, l'histologie et la sémiologie). A la fin des années 1970, les enseignants de la Faculté Mc Master (Hamilton, Ontario) ont testé un nouveau curriculum caractérisé principalement par le remplacement des cours magistraux par un auto-apprentissage en petits groupes d'étudiants sous la supervision d'un tuteur (ou apprentissage par problèmes, plus couramment appelé APP). Cette réforme pédagogique repose sur 3 concepts :

- auto-apprentissage par petits groupes d'étudiants

- orientation de l'apprentissage vers la résolution de problèmes cliniques

- évidence-based medicine (EBM) prise comme référence

Après une dizaine d'années d'adaptation et d'évolution, cette réforme, également appliquée dans d'autres facultés comme celle de Sherbrooke (Québec), a été présentée en 1992 dans une publication du JAMA [5]. Cette dernière est considérée comme l'acte fondateur de l'EBM, mais ce terme avait déjà été dévoilé par l'un des concepteurs de cette réforme, G. Guyatt, dans un article paru en 1991 dans l'ACP Journal Club [7].

En réalité, le concept d'EBM est plus ancien : on retrouve déjà cette approche dans le « critical apparaisal » (méthode de lecture critique de la littérature) et dans le développement de l'épidémiologie clinique, proposés quelques années auparavant par des auteurs américains... qui considèrent que les travaux de Pierre-Charles-Alexandre Louis (1787-1872) comportent les prémisses de l'EBM [11]. Ce médecin français préconisa l'introduction de la méthode numérique, c'est-à-dire le recours à l'épidémiologie clinique et aux statistiques. Avec cette méthode, qui rompait avec la médecine théorique non reliée aux faits pratiquée à l'époque, il démontra entre autres, en 1828, l'inutilité, voire le rôle néfaste, de l'utilisation quasi systématique des saignées et des sangsues, proposée par Broussais [10]. Cette étude fut violemment critiquée par Broussais, mais l'épidémie de choléra survenue en 1832 allait confirmer la validité de la méthode numérique de Louis. Néanmoins, malgré sa notoriété, Louis ne fut jamais admis à la Faculté de Médecine de Paris, bien qu'entre 1830 et 1850 , il fut considéré comme le plus célèbre enseignant parisien. Ses travaux, sa méthode numérique et son approche septique ont rapidement obtenu une reconnaissance internationale. Il forma de nombreux étudiants étrangers, surtout américains. En 1832, des élèves genevois (Louis-Marc D'Espine, Théodore Maunoir et John Bizot) fondèrent la Société d'observation médicale à Paris, et la même année deux élèves américains (Oliver Wendell Holmes et George Shattuck) la Society for Medical Observation à Boston [11].

Parallèlement à la réforme pédagogique, la Faculté de Mc Master a œuvré, avec d'autres facultés anglophones, pour le développement de l'épidémiologie clinique. Cette dernière allait favoriser la mise en place de réseaux internationaux et la structuration des compétences. L'EBM reprend en fait les principes de l'épidémiologie clinique... et cette dernière, dans sa version moderne, fournit la plupart des références utilisées pour l'EMB. Ceci n'est guère surprenant car on retrouve les mêmes leaders - G. Guyatt et D. Sackett - dans ces deux branches de la Médecine, à l'époque tous les deux enseignants à la Faculté Mc Master.

L'EBM, d'abord conçue comme élément d'une nouvelle méthode d'apprentissage, est devenue progressivement une (la) référence pour la pratique médicale. L'EBM - médecine factuelle ou médecine fondée sur les (niveaux de) preuve(s) ou médecine fondée sur les faits, ou encore médecine fondée sur des éléments probants pour nos confrères québécois - et l'APP pour apprentissage par problèmes (ou PBL pour problem-based learning) ont beaucoup fait parlé d'eux dans les années 1990 car, très rapidement, des avant-gardistes ou des idéologues, bien sûr zélateurs mais quelquefois doctrinaires et sectaires, ont introduit une nouvelle dialectique suscitant chez quelques uns de vives réactions. N'aurait-il pas été plus simple de présenter l'EBM comme une méthode probablement plus apodictique, plus heuristique, plus zététique... que l'approche traditionnelle, ou seulement plus assertorique. Sackett, l'un des concepteurs de l'EBM, a estimé qu'il devait intervenir pour rappeler la définition et donc les limites de l'EBM : « La médecine fondée sur des [niveaux de] preuves est l'utilisation consciencieuse et judicieuse des meilleures données actuelles de la recherche clinique dans la prise en charge personnalisée de chaque patient. » [13]. 


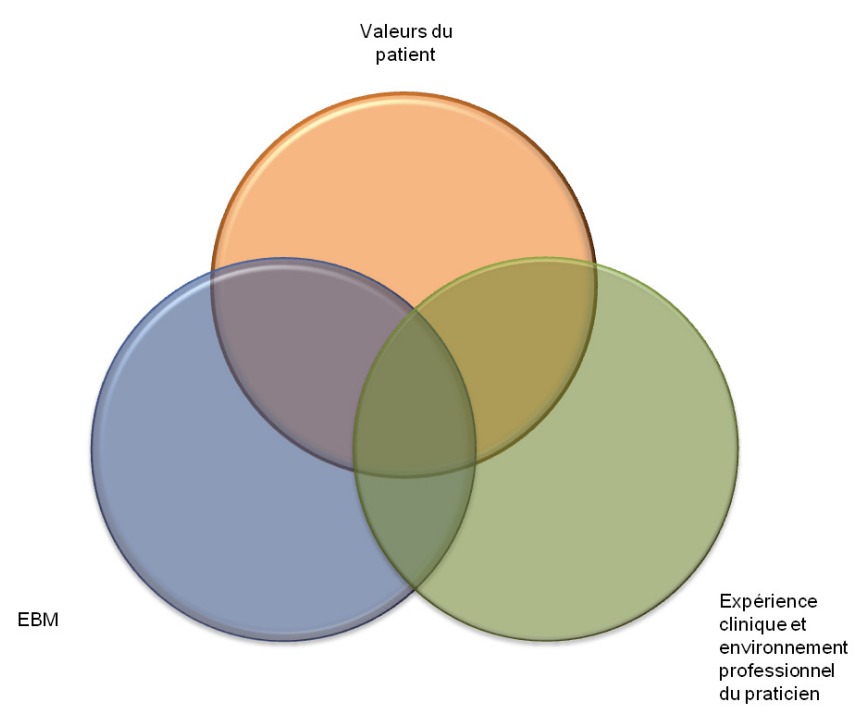

Fig. 1. Eléments intervenant dans la décision clinique.

En 2003, la définition a été de nouveau revue ( gration des meilleures données de la recherche à la compétence clinique du soignant et aux valeurs du patient. ») probablement dans le but de rassurer ceux qui suspectaient la mise en place d'une «dictature des preuves ». Par ailleurs, il faut reconnaitre qu'il était temps de penser aux droits du patient, déjà pour rester conforme à l'évolution de la législation française.

Afin de bien formaliser la place de l'EBM, Sackett et al. [13] ont a proposé un schéma constitué par 3 cercles (Fig. 1) représentant les éléments à prendre en compte lors des soins aux patients :

- les données de la recherche (les preuves)

- l'expérience clinique et l'environnement professionnel du praticien

- les valeurs ou les préférences du patient.

Certains ajoutent volontiers un $4^{\text {ème }}$ cercle (l'approche physiopathologique) (Fig. 2), sans doute pour rappeler que cet élément, souvent pertinent dans la démarche diagnostique, intervient également dans la proposition clinique finale [2]. L'intersection de ces 3 ou 4 cercles représente la zone de décision optimale.

Sur le fond, tout le monde est d'accord et personne n'oserait envisager une médecine qui ne soit pas fondée sur des faits ou sur des preuves. Mais, dans la mesure du possible, ne pratiquait-on pas déjà un peu ainsi auparavant ? L'EBM n'aurait-elle pas eu tendance à faire de nous des $M$. Jourdain ?

L'EBM a fait l'objet de nombreuses présentations et de plusieurs centaines de publications, et maintenant on connait tous la pyramide des preuves de Rosenberg et Donald [12] dont le sommet est occupé par les méta-analyses et les essais cliniques randomisés; pour être encore plus concret, il a été proposé d'attribuer un niveau de preuve aux publications,

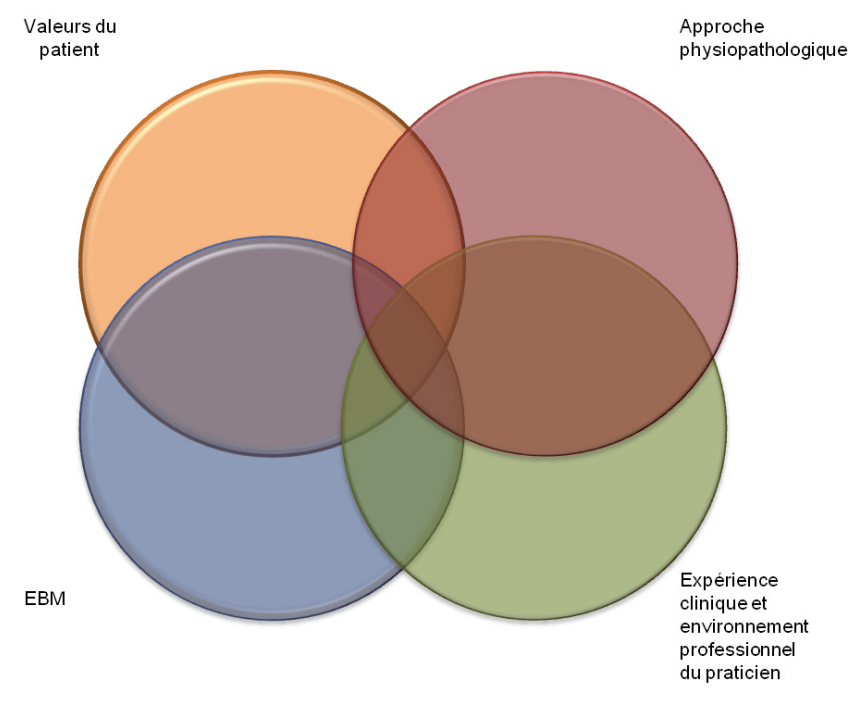

Fig. 2. Eléments intervenant dans la décision clinique.

permettant ainsi de donner un grade aux recommandations (Tab. I).

Malgré plusieurs remodelages, l'EBM continue de faire l'objet de nombreuses critiques, sans parler de ses réelles limites. On entend principalement deux critiques :

- on ne dispose pas de preuves dans de nombreux cas (surtout en Médecine bucco-dentaire) ; c'est les «zones grises » de l'EBM

- il est parfois difficile d'appliquer à un patient déterminé les données d'une méta-analyse

Nous ne poursuivrons pas davantage dans cette direction car l'analyse critique de l'EBM, des méta-analyses et des essais cliniques randomisés a déjà été faite par des personnes hautement qualifiées. Pour dresser le bilan de l'EBM après 20 ans d'application, nous allons revenir sur quelques faits marquants récents, plus ou moins médiatisés.

Prenons comme premier exemple le cas emblématique de l'infection à VIH et du SIDA. Quand l'équipe de l'Institut Pasteur, composée principalement de Jean-Claude Chermann, Françoise Barré-Sinoussi et Luc Montagnier, a découvert le virus responsable - appelé LAV pour lymphadenopathy associated virus -, elle a transmis son article à Robert Gallo qui s'était proposé de le faire publier dans la revue Science où il avait quelques pages réservées. L'article paraitra le 20 mai $1983 \ldots$ mais avec un résumé rédigé par Gallo où ce dernier transforme le nom du LAV en human T lymphotropic virus (HTLV). Il s'agit d'une traduction ambiguë qui permettra à Gallo de laisser accroire que c'est un virus comparable aux deux autres HTLV qu'il vient de découvrir. Profitant de la confusion et de quelques incertitudes sur la nature exacte du LAV, Gallo convainc rapidement les autorités scientifiques et politiques américaines qu'il est l'auteur de la découverte du VIH. Ainsi le 24 avril 1984, Margaret Heckler, Ministre de la Santé dans l'Administration 
Tableau I. Classification des preuves scientifiques (articles) et des recommandations proposée par l'HAS.

\begin{tabular}{|c|c|}
\hline Niveau de preuve scientifique fourni par la littérature & $\begin{array}{l}\text { Grade } \\
\text { des recommandations }\end{array}$ \\
\hline $\begin{array}{l}\text { Niveau } 1 \\
\text { Essais comparatifs randomisés de forte puissance } \\
\text { Méta-analyse d'essais comparatifs randomisés } \\
\text { Analyse de décision basée sur des études bien menées }\end{array}$ & $\begin{array}{l}\text { Grade } \mathbf{A} \\
\text { Preuve scientifique établie }\end{array}$ \\
\hline $\begin{array}{l}\text { Niveau } 2 \\
\text { Essais comparatifs randomisés de faible puissance } \\
\text { Etudes comparatives non randomisées bien menées } \\
\text { Etudes de cohorte }\end{array}$ & $\begin{array}{l}\text { Grade B } \\
\text { Présomption scientifique }\end{array}$ \\
\hline $\begin{array}{l}\text { Niveau } 3 \\
\text { Etudes cas-témoins }\end{array}$ & \multirow[b]{2}{*}{$\begin{array}{l}\text { Grade } \mathbf{C} \\
\text { Faible niveau de preuve } \\
\text { scientifique }\end{array}$} \\
\hline $\begin{array}{l}\text { Niveau } 4 \\
\text { Etudes comparatives comportant des biais importants } \\
\text { Etudes rétrospectives } \\
\text { Séries de cas } \\
\text { Etudes épidémiologiques descriptives (transversale, longitudinale) }\end{array}$ & \\
\hline
\end{tabular}

du Président Reagan, annonce lors d'un show télévisé la découverte faite par Gallo. L'Institut Pasteur s'en offusque et porte plainte. Un accord amiable, paraphé par les Présidents Reagan et Chirac, sera proposé pour mettre un terme au conflit; la muflerie de Gallo aura permis aux Etats Unis de bénéficier de $50 \%$ des royalties provenant de l'exploitation des tests de dépistage. Par la suite, le gouvernement américain a néanmoins diligenté une enquête confiée à l'OSI (Office of Science Intregrity). Au terme de cette enquête, Gallo a été prié de démissionner du NIH (National Institute of Health)... ce qui ne l'empêchera pas de s'étonner quelques années plus tard de ne pas avoir reçu le prix Nobel en 2008 avec deux des trois découvreurs français du VIH [4].

Au sujet de l'infection à VIH, il y a une autre péripétie que l'on peut méditer. Avant l'apparition de cette infection, on pratiquait systématiquement un BW (réaction de BordetWassermann) dans différentes occasions (hospitalisation, mariage, grossesse) et, devant toute sérologie syphilitique positive, on devait réaliser une enquête épidémiologique et obliger le(s) sujet(s) séropositif(s) à se faire soigner, éventuellement en recourant à la force publique. C'était l'époque où l'intérêt général primait sur l'intérêt individuel. Avec l'apparition de l'infection à VIH, le principe du dépistage systématique d'une maladie contagieuse fut abandonné car il était considéré comme antinomique avec le respect de la liberté individuelle. Et il était malvenu de s'en offusquer même lorsqu'on disposât d'un traitement réellement efficace contre le VIH. Plus de 25 ans après l'apparition de l'infection à VIH, on est bien obligé d'admettre que les mesures préventives sont insuffisantes (en France, le nombre de contamination augmentent chaque année et environ 50000 personnes ignoreraient leur séropositivité ou ne seraient pas suivies). Ce constat a amené à un changement de paradigme : diverses recommandations incitent désormais à banaliser le dépistage et « le souhait de réduire le risque de transmission du VIH peut donc désormais constituer un argument recevable pour l'initiation d'un traitement antirétroviral »[3]. Était-il nécessaire d'attendre si longtemps pour accepter ce qui, en toute logique, paraissait évident ?

Les revues scientifiques disposent d'un comité de rédaction et d'un comité de lecture pour évaluer les articles soumis à publication. Régulièrement, leur partialité est dénoncée et certaines revues apparaissent même sous la coupe de potentats qui régentent la politique éditoriale, se réservant le droit de modifier le résumé ou les conclusions d'un article, ou de publier l'article d'un protégé, et bien sûr de refuser éventuellement un article quand les auteurs ne font pas partie de la nomenklatura. Prenons deux exemples pour illustrer cette situation. En 1998, Wakefield et al. ont publié dans le Lancet un article suggérant qu'il existerait un lien entre le vaccin contre la rougeole, les oreillons et la rubéole (ROR) et la survenue d'une hyperplasie lymphoïde iléale, d'une colite et d'une forme d'autisme [14]. Cet article eut un retentissement important et il fut largement diffusé par ceux qui suspectent systématiquement les vaccins d'être à l'origine de bien des maux. C'est un journaliste spécialisé, Brian Deer, qui a découvert la fraude et publié son enquête dans le Sunday Times en 2004. Après plusieurs années d'enquête, Wakefield et WalkerSmith, les deux principaux auteurs, ont été radiés en mai 2010 du registre des médecins britanniques par le General Medical Council, le Lancet a retiré cet article responsable d'une psychose anti-ROR (rétractation partielle en février 2004, complète en février 2010) et, dans son numéro du 5 janvier 2011, 
le British Medical Journal accuse Wakefield d'avoir délibérément falsifié les résultats de son étude.

Autre exemple où le comité de rédaction et le comité de lecture ont manqué de vigilance. Dans le numéro de décembre 2010 de Oral Surgey, Oral Medicine, Oral Pathology, Oral Radiology, and Endodontology, on peut lire un article sur l'attitude à adopter pour des extractions dentaires chez les patients sous antivitamines $\mathrm{K}$ [1]. Après un examen sommaire, cet article fait toujours illusion car on y trouve tous les ingrédients d'un article «moderne » : arbre décisionnel, statistiques... Mais très vite plusieurs questions surgissent. Il est inhabituel qu'un article publié dans cette revue soit signé par un seul auteur. De plus, cet auteur n'a aucune activité universitaire : c'est un confrère omnipraticien de Vancouver (Canada), probablement très compétent en informatique (il est le webmaster d'une association professionnelle). Il a découvert l'existence d'un logiciel, le TreeAge Pro 2009 (TreeAge Software, Willamstow, MA), qui permet d'analyser statistiquement les différentes branches de l'arbre. Il l'a d'abord utilisé pour la prise en charge des caries occlusales, et il en a fait un article. Il récidive en essayant de définir l'attitude à adopter pour réaliser les extractions dentaires chez les patients sous antivitamines $K$. Le résultat est ubuesque : que peut faire un praticien de toutes ces données statistiques ? Par ailleurs, on est interloqué de voir l'auteur exposer benoîtement d'abord les références qui lui ont permis de faire un peu mieux connaissance avec la physiopathologie cardiaque, et citer seulement une dizaine d'articles concernant directement le sujet de son article. $0 \mathrm{n}$ est loin d'une revue conséquente de la littérature qui tenterait de suppléer l'absence de méta-analyse sur le sujet. Dans ces conditions, quelle valeur peut-on accorder au risque relatif et à la valeur du $p$ donnés à chaque situation ? Au total, cet article constitue un simulacre de publication scientifique. L'arbre décisionnel, à la mode depuis une vingtaine d'années, fonctionne sur un mode binaire, donc manichéen, qui ne correspond pas à la réalité de la pathologie médicale. A l'heure de l'intelligence artificielle, on peut s'étonner qu'un auteur aussi féru d'informatique puisse ignorer les travaux du Laboratoire d'intelligence artificielle et d'informatique mis au point par le MIT (Massachussetts Institute of Technology). Ce laboratoire a développé, entre autres, Exhibit qui permet, avec le système de facettes, d'effectuer une démarche diagnostique beaucoup plus sophistiquée que celle pratiquée avec un arbre décisionnel.

Après l'histoire du vaccin $\mathrm{H}_{1} \mathrm{~N}_{1}$, les laboratoires pharmaceutiques sont de nouveau sur la sellette. L'enquête sur le Médiator $^{\circledR}$ a révélé (confirmé diront certains) l'existence de graves dysfonctionnements au sein de l'AFSSAPS et des différentes instances chargées de la mise sur le marché des médicaments, de l'évaluation du service médical rendu (SMR) et de son amélioration (ASMR), de leurs effets indésirables (Commission nationale de pharmacovigilance)... Dans son dernier rapport, l'IGAS (Inspection générale des Affaires sociales) n'hésite pas à parler
« d'incompréhensible tolérance ». Les hommes politiques s'offusquent à la lecture de ce rapport et semblent découvrir que le système français du médicament est gangréné. Les ministres responsables de la Santé n'auraient jamais été informés par l'AFSSAPS. C'est peut-être vrai mais quel manque de vigilance et quelle inconséquence ? Pourquoi n'ont-ils pas écouté ceux qui dénoncent le système depuis plusieurs années [9] ?

On ne peut pas évoquer le Médiator ${ }^{\circledast}$ sans dire quelques mots de l'Isoméride, son demi-frère ainé. Il a été retiré du commerce en 1997 car il créait des lésions des valves cardiaques. Il y a eu environ 20000 victimes aux Etats-Unis et la procédure judiciaire a abouti à une indemnisation de 3,75 milliards de dollars [6]. En France, deux victimes seulement auraient été indemnisées (environ $418000 €$ pour l'une et $145000 €$ pour l'autre) après 10 ans de procédure! Cet effet indésirable, ces procès et le retrait du marché du Médiator ${ }^{\circledR}$ en Suisse, Espagne, Italie auraient dû alerter le laboratoire pharmaceutique et lui faire envisager le retrait définitif du Médiator. En France, pour prendre la décision du retrait du marché, il faudra attendre une dizaine d'années malgré plusieurs interventions restées sans suite :

- Septembre 1998 : les professeurs Hubert Allemand (CNAMTS), Claudine Blum-Boisgard (CANAM) et Patrick Choutet (Mutuelle sociale agricole) adressent une lettre à Jean-René Brunetière, directeur général de l'Agence du médicament, concernant le Médiator ${ }^{\circledR}$ et ils écrivent entre autres : "Il nous apparaîtrait opportun de procéder à une réévaluation de l'utilité du Médiator ${ }^{\circledR}$ dans la stratégie thérapeutique de la maladie diabétique et dans celle des hyperlipidémies ».

- 2001 : le Médiator ${ }^{\circledast}$ fait partie de la liste, rendue publique, des 800 médicaments proposés au déremboursement pour SMR insuffisant. Après quelques négociations, le Médiator ${ }^{\circledR}$ retrouve son SMR initial et conserve son taux de remboursement maximal de $65 \%$.

- 2005 : vive polémique à propos du Médiator ${ }^{\circledast}$ entre Jean Marimbert, directeur de l'AFSSAPS et la revue Prescrire, après l'éditorial « L'Agence française des produits de santé est-elle avant tout au service des patients, ou au service des firmes pharmaceutiques?»

- Juin 2006 : François Autin, sénateur et médecin, obtient l'ouverture d'une mission d'information sur « les conditions de mise sur le marché et de suivi des médicaments »; le rapport final est intitulé « Restaurer la confiance » ?

- Mai 2008 : Catherine Lemorton, députée et pharmacienne, remet un rapport sur « La prescription, la consommation et la fiscalité des médicaments ».

Ce rapport et le précédent qui s'intéressent à l'industrie pharmaceutique et à ses méthodes, sont restés sans suite. Ils étaient pourtant accompagnés respectivement de 25 et 92 propositions pour réformer les organismes s'occupant des médicaments et apporter un peu plus de transparence dans leur fonctionnement. 
- Février 2009 : Cristiana Muscarisini, députée italienne, interpelle la Commission européenne à propos du Médiator ${ }^{\circledR}$.

- Juin 2009 : documentaire "Les médicamenteurs » sur France 5 le 9 juin 2009 ; rediffusion sur LCP Public Sénat, le 22 janvier 2011.

L'investissement et la pugnacité d'Irène Frachon font enfin plier l'AFSSAPS : cette dernière ordonne le retrait effectif du Médiator ${ }^{\circledast}$ du marché français pour le 30 novembre 2009. En janvier 2011, l'IGAS publie son rapport : il rend officiel ce qui avait été dénoncé dans les rapports F. Autin et C. Lemorton, par la revue Prescrire, par le Canard enchaîné...

L'HAS et l'AFSSAPS comportent de nombreuses instances, commissions... où siègent des médecins compétents qui ont sans doute entendu parler de l'EBM. Qu'en ont-ils fait? Enfin, notons que, comme pour le distilbène, c'est une étude cas-témoins (étude de niveau 3 selon la classification de l'HAS) qui a apporté la preuve de la toxicité du Médiator ${ }^{\circledR}$.

Peut-on espérer un jour arriver à la séparation de « la lumière d'avec les ténèbres » ? L'EBM constitue sans doute un bon moyen pour y arriver mais, manifestement, il n'est pas suffisant. Aux cercles proposés pour formaliser l'EBM, il faudrait sans doute rajouter un cercle, un cercle vertueux, pour rappeler l'importance de l'éthique médicale et des conflits d'intérêt.

On peut donc penser à postériori qu'il était manifestement présomptueux d'imaginer que quelques recettes suffiraient à faire évoluer la pratique médicale et les médecins. Sans doute les promoteurs de l'EBM n'avaient pas lu Fontenelle : "Nous qui sommes hommes, ne savons-nous pas bien jusqu'à quel point d'autres hommes ont pu être ou imposteurs ou dupés? ». Sinon ils auraient sans doute été plus prévoyants.

Conflits d'intérêt : aucun

\section{Références}

1. Balevi B. Should warfarin be discontinued before a dental extraction ? A decision-tree analysis. Oral Surg Oral Med Oral Pathol Oral Radiol Endod 2010;110:691-7.

2. Cornuz J, Bischof T. Evidence-based medicine : nécessaire, mais pas suffisant. Med Hyg 2004;62:2339-40.

3. Deleuze J. Prévention de l'infection par le VIH : changer de paradigme. Rev Prat 2010;60:1335.

4. Documentaire sur France 2. La bataille du SIDA. 25 novembre 2010.

5. EBM working group. Evidence-based medicine : a new approch to the teaching of medicine. JAMA 1992;268:2420-5.

6. Frachon I. Mediator $150 \mathrm{mg}$. Combien de morts ? Editions Daialogues.fr, Brest, 2010.

7. Guyatt G. Evidence-based medicine. ACP J Club 1991;114:A16.

8. Haynes R, Devereaux P, Guyatt G. Clinical expertise in the era of evidence-based medicine and patient choice. ACP J Club 2002;136:A11-A14.

9. Horel S. Les médicamenteurs. Editions du Moment, Paris, 2010.

10. Louis PCA. Recherche sur les effets de la saignée dans plusieurs maladies inflammatoires. Arch Gen Med 1828;18:321-36.

11. Morabia A. Pierre-Charles-Alexandre Louis and the birth of clinical epidemiology. J Clin Epidemiol 1996;499:1327-33.

12. Rosenberg W, Donald A. Evidence based medicine: an approach to clinical problem-solving. Br Med J 1995;310:1122-6.

13. Sackett DL, Rosenberg WMC, Gray JA, Haynes RB, Richardson WS. Evidence based medicine: what it is and what isn't. Br Med J 1996;312:71-2.

14. Wakefield AJ, Murch SH, Anthony A, Linnell J, Casson DM, Malik M, Berelowitz M, Dhillon AP, Thomson MA, Harvey P, Valentine A, Davies SE, Walker-Smith JA. Ileal-lymphoid-nodular hyperplasia, non-specific colitis, and pervasive developmental disorder in children. Lancet 1998;351:637-41. 